\title{
A Numerical Tool for Transmission Lines
}

\author{
Hervé Bolvin, André Chambarel, and Philippe Neveux \\ UMR A114 Climate, Soil and Environment \\ 33 rue Louis Pasteur, F-84000 Avignon, France
}

\begin{abstract}
The electric line numerical study is usually made through a harmonic approach with the impedance concept based on the Finite Difference discretization in both the time and space domains. These methods present severe drawbacks when used in impulse working and space dependent parameters. We present here an efficient numerical tool for electric line simulation. The mathematical model is based on the telegrapher's equations. In the present paper, we propose a new approach based on the Finite Element Method associated with an efficient algorithm for the numerical resolution of the telegrapher's equations. In practice the electric line is connected to the entrance and the exit with linear or non linear circuits. The objective of this paper is to provide an efficient numerical tool for electric line simulation in complex configurations.
\end{abstract}

Keywords: Transmission line, finite element method, differential system, impulse voltage, stiff time problem.

\section{Introduction}

The electric line model shall be built in order to analyze propagation phenomena [1]. Usually the transmission lines are connected at both ends to other circuits, the supply circuit at the entrance and the utilization circuit at the exit (Fig.1). As a matter of fact, the analysis of the recorded output signal leads to the characterization of the line. Hence, the identification of line properties can be done by coupling this model with an optimization scheme [2][3].

The propagation equations are Partial Differential Equations (PDE) both in time and space. In order to solve the wave propagation problem, various approaches have been developed. Many authors use a harmonic approach sometimes associated with a numerical method for space discretization [4]. This method is generally uneasy to use particularly in impulse working with wave fronts and space dependent electric properties. Moreover it is not available in the case of non linear connected circuits or non linear electric properties. Also, the numerical approach of the problem in the time and space domain is essentially based on the FDTD method [5]. For these reasons we propose a general numerical tool for the simulation of the transmission line for a large class of configurations, based on the Finite Element Method (FEM) for space discretization, and a Modified Backward Difference Method (MBDM) [6] for time discretization. The FEM allows complex boundary conditions to be considered [7], and the MBDM is well recommended for stiff time problems. 


\section{Statement of the Problem}

Let us consider a transmission line with entrance and exit circuits ( Fig. 1).

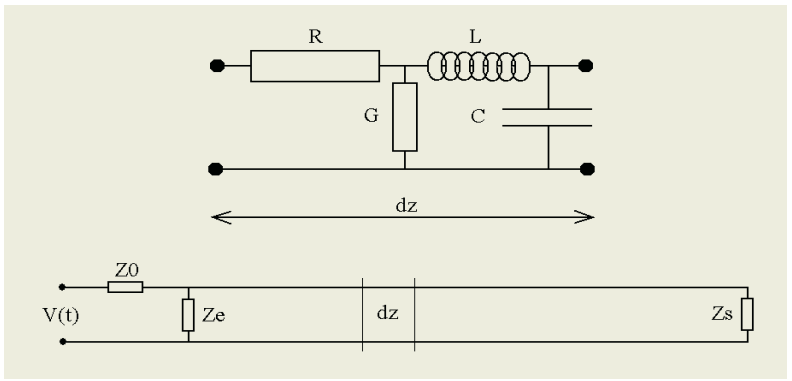

Fig. 1. The electric line pattern

We obtain the following dimensionless model :

$$
\begin{array}{lll}
\frac{\partial i^{\prime}}{\partial z^{\prime}}=-C^{*} \cdot \frac{\partial v^{\prime}}{\partial t^{\prime}}-G^{*} \cdot v^{\prime} & & \mu_{r} \cdot \frac{\partial i}{\partial t}=-\frac{\partial v}{\partial z}-a \cdot i \\
\frac{\partial v^{\prime}}{\partial z^{\prime}}=-L^{*} \cdot \frac{\partial i^{\prime}}{\partial t^{\prime}}-R^{*} \cdot i^{\prime} & \rightarrow & \varepsilon_{r} \cdot \frac{\partial v}{\partial t}=-\frac{\partial i}{\partial z}-b \cdot v
\end{array}
$$

We can propose a technique capable of approaching complex boundary conditions:

- At the entrance of the line we can apply a time dependent voltage, and the propagation phenomenon takes place in an open transmission line.

- We can include the electric characteristics of the voltage source with its connection at the entrance and a known impedance at the exit. This circuit possibly contains resistances, capacitances and self-inductions.

\section{Finite Element Formulation}

We have tested a new approach by the FEM for space discretization. Here the advantage of the FEM concerns the boundary conditions and the space-dependent electric properties. In fact we have natural boundary conditions and essential boundary conditions [8][9]. In accordance with these boundary conditions different Finite Element formulations are possible. So the corresponding boundary conditions can be expressed through Dirichlet's conditions or mixed conditions.

\subsection{Weak Formulations}

The Galerkin weighting applied to the system (1) gives rise to a weak formulation. In accordance with the boundary conditions of the problem under consideration, one can use one of the following formulations (2) (3) obtained via different integrationby-parts schemes; we present examples : 


$$
\begin{aligned}
& \left\{\begin{array}{l}
\int_{0}^{1} \delta i \cdot \mu_{r} \cdot \frac{\partial i}{\partial t} \cdot d z=-\int_{0}^{1} \delta i \cdot \frac{\partial v}{\partial z} \cdot d z-\int_{0}^{1} \delta i \cdot a \cdot i \cdot d z \\
\int_{0}^{1} \delta v \cdot \varepsilon_{r} \cdot \frac{\partial v}{\partial t} \cdot d z=-\int_{0}^{1} \delta v \cdot \frac{\partial i}{\partial z} \cdot d z-\int_{0}^{1} \delta v \cdot b \cdot v \cdot d z
\end{array}\right\} \\
& \left\{\begin{array}{l}
\int_{0}^{1} \delta i \cdot \mu_{r} \cdot \frac{\partial i}{\partial t} \cdot d z=-[\delta i \cdot v]_{0}^{1}+\int_{0}^{1} v \cdot \frac{\partial(\delta i)}{\partial z} \cdot d z-\int_{0}^{1} \delta i \cdot a \cdot i \cdot d z \\
\int_{0}^{1} \delta v \cdot \varepsilon_{r} \cdot \frac{\partial v}{\partial t} \cdot d z=-\int_{0}^{1} \delta v \cdot \frac{\partial i}{\partial z} \cdot d z-\int_{0}^{1} \delta v \cdot b \cdot v \cdot d z
\end{array}\right\}
\end{aligned}
$$

These formulations are mathematically equivalent. We can choose the one that best fits the form of the model of boundary conditions.

\subsection{Matricial Formulations}

For the implementation of the different formulations (2) (3) for users, we must be aware of the details of matricial formulation. For this reason we have to provide the significant example below. Line length is discretized into $n_{e}$ elements $\left(\Omega_{\mathrm{e}}\right)$ with . $\bigcup_{e}\left(\Omega_{e}\right)=(\Omega)$. We have used Lagrange's linear polynomial base $<\mathrm{n}(\mathrm{z})>[7]$. We have choosen the first formulation (2) to illustrate the matricial form. The geometrical and analytical discretizations can be written as follows :

$$
\begin{aligned}
& \sum_{n e}\left(\left\langle\delta i_{e}, \delta v_{e}\right\rangle \cdot \int_{\left(\Omega_{e}\right)}\left[\begin{array}{cc}
\{n\} \cdot \mu_{r} \cdot\langle n\rangle & 0 \\
0 & \{n\} \cdot \varepsilon_{r} \cdot\langle n\rangle
\end{array}\right] \cdot d z \cdot\left\{\begin{array}{c}
\frac{\partial i_{e}}{\partial t} \\
\frac{\partial v_{e}}{\partial t}
\end{array}\right\}\right)= \\
& \sum_{n e}\left(\left\langle\delta i_{e}, \delta v_{e}\right\rangle \cdot \int_{\left(\Omega_{e}\right)}\left[\begin{array}{cc}
-\{n\} \cdot a \cdot\langle n\rangle & -\{n\} \cdot\left\langle\frac{\partial n}{\partial z}\right\rangle \\
-\{n\} \cdot\left\langle\frac{\partial n}{\partial z}\right\rangle & -\{n\} \cdot b \cdot\langle n\rangle
\end{array}\right] \cdot d z \cdot\left\{\begin{array}{c}
i_{e} \\
v_{e}
\end{array}\right\}\right)
\end{aligned}
$$

The general matricial formulation can be summarized under the form:

$$
\sum_{n e}\left(\left\langle\delta u_{e}\right\rangle \cdot\left[m_{e}\right] \cdot\left\{\frac{\partial u_{e}}{\partial t}\right\}\right)=\sum_{n e}\left\langle\delta u_{e}\right\rangle \cdot\left(\left\{f_{e}\right\}-\left[k_{e}\right] \cdot\left\{u_{e}\right\}\right)
$$

where index $e$ denotes the element number and : $\quad\left\langle\mathrm{u}_{\mathrm{e}}>=<\mathrm{i}_{\mathrm{e}}, \mathrm{v}_{\mathrm{e}}>\right.$ After the assemblage process we obtain the following differential system :

$$
[M] \cdot \frac{\mathrm{d}}{\mathrm{dt}}\{U\}=\{\mathrm{F}\}-[\mathrm{K}] .\{\mathrm{U}\} \text { where }\{\mathrm{U}\}=\left\{\mathrm{i}^{(\mathrm{G})}, \mathrm{v}(\mathrm{G})\right\}
$$

Index $\mathrm{G}$ denotes the global space. 


\section{Numerical Method}

\subsection{The Modified Backward Difference Method Technique (MBDM)}

For all weak formulations, the general formulation of the differential system is [8] :

$$
\frac{d}{d t}\{U\}=\{\Phi(\mathrm{U}, \mathrm{t})\} \quad \text { where }\{\Phi(U, t)\}=[\mathrm{M}]^{-1} \cdot(\{\mathrm{F}\}-[\mathrm{K}] \cdot\{\mathrm{U}\})
$$

For the numerical time-resolution, the MBDM is used. With the proposed formulation one can choose the time order of discretization and the upward scheme [6][10]. In this context, it is possible to apply a matrix-free technique. As a matter of fact the mass matrix and the stiffness matrix are never built during the calculation. As a consequence, we obtain a high performance level in terms of CPU time and storage cost. This technique can be summarized in the following algorithm :

$$
\begin{aligned}
& t_{n}=0 \\
& \text { while } \quad\left(t_{n} \leq t_{\max }\right) \\
& \qquad\left\{\begin{array}{r}
\left.\left\{\Delta U_{n}^{i}\right\}=\Delta t_{n} \cdot \sum_{j=0}^{k-1} \lambda_{j} \cdot\left[M_{n-j}^{i}\right]^{-1} \cdot\left\{\Psi_{n-j}\left(U_{n-j}+\alpha_{j} \cdot \Delta U_{n-j}^{i-1}, t_{n}+\alpha_{j} \cdot \Delta t_{n}\right)\right\}\right\} \\
i=1,2, \ldots \text { until }\left\|\Delta U_{n}^{i}-\Delta U_{n}^{i-1}\right\| \leq \text { tolerence }
\end{array}\right\} \\
& \qquad \begin{array}{r}
\left.U_{n+1}\right\}=\left\{U_{n}\right\}+\left\{\Delta U_{n}\right\} \\
t_{n+1}=t_{n}+\Delta t_{n}
\end{array} \\
& \text { end while }
\end{aligned}
$$

\subsection{The Software}

Fig. 2 shows the general structure of the compact code. It is organized in three classes corresponding to the functional blocks of the FEM's different stages [11].

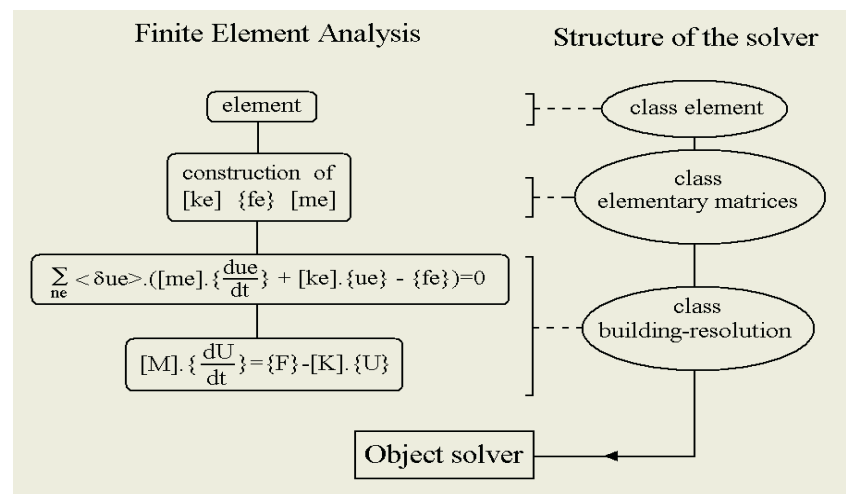

Fig. 2. Object structure of a standard solver 


\section{Numerical Results}

\subsection{The Benchmark}

Let us consider a transmission line with an open circuit at the exit. So we have Dirichlet conditions at both ends of the line. In this case, weak formulation (2) before the discretization of the mathematical model is the most convenient one.

In order to test the proposed method, a very unfavorable case is considered : line without loss, small space-discretization (1000 elements), first order timediscretization and time discontinuity as initial condition (step of voltage).

The main difficulty of the numerical computation of the wave front propagation is that numerical instabilities may occur. The MBDM technique provides an upward time-parameter $\alpha$. The numerical instability control has been calculated for different values of parameter $\alpha$ : thus we can determine an optimal value for $\alpha$.

Fig. 3 shows the time dependent intensity signal at the entrance of the line and Fig. 4 , the space dependent intensity signal at $t=0.5$.
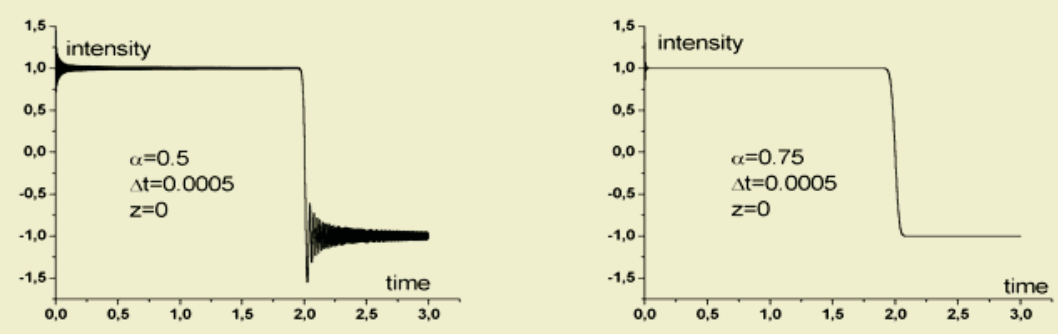

Fig. 3. Time dependent intensity at the entrance of the line for two values of $\alpha$

One can notice from Fig. 3 and Fig. 4, that if the $\alpha$-value is lower than 0.5 , no stable numerical solution can be obtained with a realistic time step. By using an optimal value of $\alpha$, we obtain the well-known signal with a rectangular form (Fig.5).
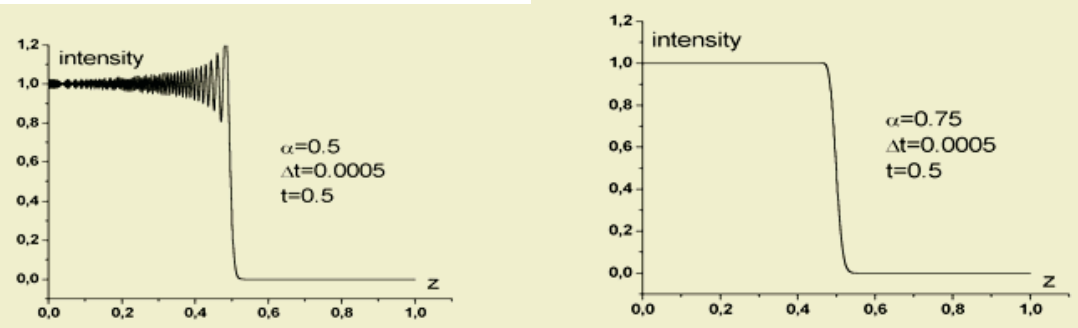

Fig. 4. Space dependent intensity at $t=0.5$ for different values of $\alpha$ 


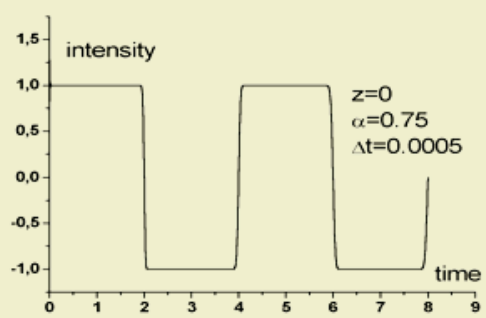

Fig. 5. Time dependent intensity at the entrance $(z=0)$ of the line

\subsection{Example of a Non Linear Circuit}

Let us examine the case of a non linear circuit (a transistor, for example) connected at the end of the line. Fig. 6 presents a bipolar transistor.

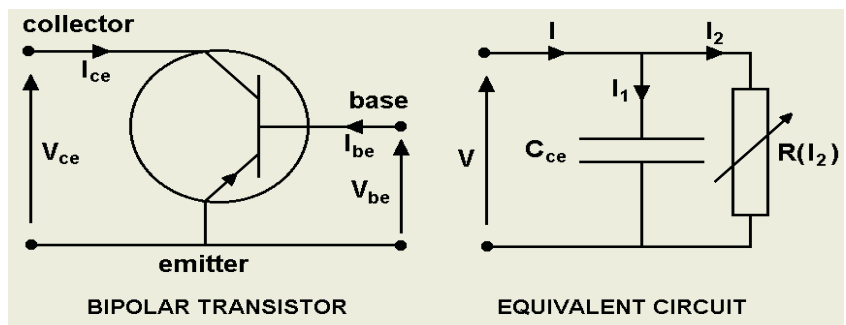

Fig. 6. An example of non linear circuit

We will not give all working details about this classical electronic component. In our context the peculiarity of the problem is the non-linearity of the static currentvoltage characteristic of the collector-emitter.

For a given base-emitter voltage, the bipolar transistor can be equivalent to the circuit presented in Fig. 6. At a high frequency the collector-emitter capacitance $\mathrm{C}_{\mathrm{ce}}$ is not negligible and the static part of the transistor's characteristic is represented by a variable resistance. The dimensionless form of the intensity can be written:

$$
i=A \cdot \frac{\partial v}{\partial t}+\varphi(v)
$$

The extraction of $\varphi(v)$ in equation (9) is performed by an elementary numerical method. In impulse working this circuit is modelized by a differential equation (9). In that case we also choose formulation (3) because it is possible to introduce the intensity in the integration-by-parts directly. The voltage at the entrance is similar to the example above. At the exit of the line the term of integration-by-parts of formula (3) can be written :

$$
[\delta v \cdot i]_{0}^{1}=\left\langle\delta v_{n}\right\rangle \cdot\{n(z)\} \cdot\left(A \cdot\langle n(z)\rangle \cdot\left\{\frac{\partial v_{n}}{\partial t}\right\}+\varphi\left(v_{n}\right)\right)_{z=1}
$$


This matricial formulation is modified because we do not have here a modification of the elementary matrix $\left[\mathrm{k}_{\mathrm{e}}\right]$ but a modification of elementary electric loading $\left\{\mathrm{f}_{\mathrm{e}}\right\}$. Function $\varphi(v)$ must be built at each iteration of algorithm (8). Under these conditions and for the last element $(z=1)$ the elementary matrices concerned are modified as follows :

$$
\begin{aligned}
& \left\{\begin{array}{l}
f_{i} \\
f_{v}
\end{array}\right\}_{\text {new }}=\left\{\begin{array}{l}
f_{i} \\
f_{v}
\end{array}\right\}_{\text {old }}+\left\{\begin{array}{c}
0 \\
\{n\} . \varphi\left(v_{n}\right)
\end{array}\right\} \\
& {\left[\begin{array}{cc}
m_{i i} & 0 \\
0 & m_{v v}
\end{array}\right]_{\text {new }}=\left[\begin{array}{cc}
m_{i i} & 0 \\
0 & m_{v v}
\end{array}\right]_{\text {old }}+\left[\begin{array}{cc}
0 & 0 \\
0 & \{n .\} . A .\langle n\rangle
\end{array}\right]}
\end{aligned}
$$

Algorithm (8) is performed with the same numerical characteristics.

In Fig. 7, we notice a transient complex intensity signal at the entrance of the line. If the dimensionless time is lower than 2 a step of intensity propagates along the line, reflects at the end and comes back. After this we can see the influence of the exit circuit.

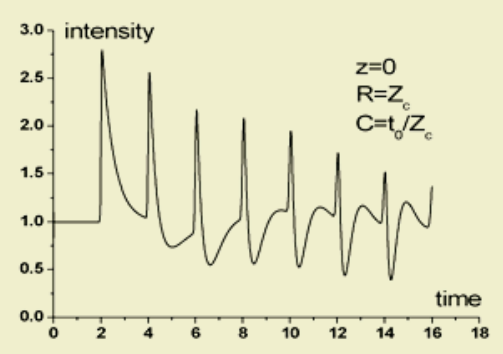

Fig. 7. Time dependent intensity at the entrance of the line in the case of a non-linear circuit connected at the exit

\section{Conclusion}

For the simulation of the transmission lines, we wish to place at the disposal of electronics specialists, a numerical tool which is effective as much by its simplicity as by its performances. The main applicability of this technique concerns complex cases of line models, these potentialities being associated to a simple data-processing tool that can be used by non specialists. By its various formulations, the method makes it possible to approach the whole of the problems quoted above. Moreover, in this context, we have developed a method of numerical resolution specific to the problems of transmission lines. We have sought significant examples which illustrate the principle of the method as well as concrete application cases.

Acknowledgement. The authors would like to thank Ralph Beisson for his assistance with the English composition of this paper. 


\section{References}

1. Magnusson P.G., Alexander G.C. and Tripathi V.K.: Transmission Lines and Waves Propagation. 3 rd Edition CRC, Boca Raton

2. Norgren M. and He S.: An Optimization Approach to the Frequency Domain Inverse Problem for a Non-Uniform LCRG Transmission Line. IEEE Transactions on Microwave Theory and Techniques, Vol. 44 (8) (1996) 1503-1507

3. Lundstedt J. and He S.: A Time-Domain Optimization Technique for the Simultaneous Reconstruction of the Characteristic Impedance, Resistance and Conductance of a Transmission Line. Journal of Electromagnetic Waves and Applications, Vol.10 (4) (1996) 581-602

4. Heimovara T.J.: Frequency Domain Analysis of Time Domain Reflectometry Wave Form, Measurement of the Complex Dielectric Permittivity of Soils. Water Resource Research, Vol. 30 (1994) 189-199

5. Hu J.-L., Chan C.H.and Sarkar T. K.: Optimal Simultaneous Interpolation / Extrapolation Algorithm of Electromagnetic Responses in Time and Frequency Domains. IEEE Transactions on Microwave Theory and Techniques, Vol. 49 (10) (2001) 1725-1732

6. Anderson D.A., Tannehill J.C. and Pletcher R.H.: Computational Fluid Mechanics and Heat Transfer. Hemisphere Publishing Corporation Editor,1984.

7. Dhatt G. and Touzot G.: Une Présentation de la Méthode des Eléments Finis (in French). Editions Maloine S.A., Paris (1981)

8. Chambarel A. and Ferry E.: Finite Element Formulation for Maxwell's Equations with Space Dependent Electric Properties. Revue Européenne des Eléments Finis, Vol. 9 (8) (2000) 941-967

9. Assous F., Degond P., Heintze E., Raviard P.A.. and Segre J.: On a Finite Element Method for Solving the Three-Dimensional Maxwell Equations. J. Comp. Phys., Vol.109 (1993) 222-237

10. Sod G.A.: A survey of Several Finite Difference Methods for Systems of Nonlinear Hyperbolic Conservation Laws. J. Comp. Phys., Vol. 27 (1978) 1-31

11. Chambarel A. and Fougère D.. A General Parallel Computing Approach using the Finite Element Method and the objects oriented programming by selected data technique, Lecture Notes in Computer Science, Vol. 2127 (2001) 428-435

\section{Nomenclature}
$R^{*}$ lineic resistance
$L^{*} \quad$ lineic inductance
$C^{*} \quad$ lineic capacitance
$\mathcal{E}_{r} \quad$ relative electric permittivity
$\mu_{r} \quad$ relative magnetic permeability
$(\Omega)$ integration domain
$\left(\Omega_{e}\right)$ element $e$

$\left[\mathrm{m}_{\mathrm{e}}\right] \quad$ elementary mass matrix

$\left[\mathrm{k}_{\mathrm{e}}\right]$ elementary electric matrix

$\left\{\mathrm{f}_{\mathrm{e}}\right\} \quad$ elementary electric loading

$\alpha \quad$ upward time-parameter

〈.) line matrix

\{.\} column matrix 